\title{
REVIEW PAPER ON HARVESTING ENERGY USING PIEZOELECTRIC TRANSDUCERS
}

\author{
Niharika Wakchaure, Shashank Waghmare, Ruchira Rakshe, Minaxi Rai Sharma, Ashish Joshi
}

\begin{abstract}
A life of human beings is dependent on the energy which they consume for their everyday purpose, it can be for illumination, running a gadget and many other purposes which contribute towards the development of mankind and also for economic growth. If global energy consumption is taken into consideration, every resource has its own quantity. As the years pass by, one can observe the depletion of naturally available conventional resources of energy. Hence, the world has been shifting to renewable energy sources such as solar, wind, tidal power. Even though, there isn't any greater transformation in the energy consumption from the conventional sources due to their higher efficiency deliveries. If an alternative energy resource has to be discovered, one has to look for the sources available in the surrounding. When the road traffic is considered for an instance, the number of vehicles travelling are enormous and due to the movement, every vehicle induces a certain number of vibrations into the pavement below which collectively turns out to be plenty if the vibrations from all the vehicles are to be taken in account. The researchers have come up with a technology to harness this energy and make it usable to the consumers. To convert one form of energy to another, a transducer can be used which is capable of transforming the entire input energy into an output voltage. This form of output is said to be called 'piezoelectricity' where the incident pressure is potentially converted into electricity that can be utilized for numerous purposes.
\end{abstract}

Keywords: Renewable energy, Alternative energy resources, Road Traffic, Piezoelectricity

\section{INTRODUCTION}

India has a road network of 58,97,671 kilometres. Out of this, approximately $2 \%$ are national highways, $3 \%$ are state highways and $10 \%$ are urban roads. The Indian automobile industry currently manufactures $26 \mathrm{MN}$ vehicles. With increasing vehicular traffic, it will be prudent to implement technology that will harvest the vibrational energy from the vehicular traffic.

Energy harvesting technologies are being studied across the globe (Park et.al. 2017). In recent years, interest in energy harvesting through transduction is increasing (Pappagiannakis et.al. 2016). Piezoelectric transducers convert mechanical energy from vibrations to electrical energy. Vehicle vibration can not only cause damage to the road but also lead to a waste of energy (Xu et.al. 2017).

This review paper investigates various methods used for harvesting the vibrational energy from the vehicles and utilizing that to produce electricity. The purpose of this review paper is to identify the research gaps and opportunities in utilizing piezoelectric pads for the generation of electricity.

\section{LITERATURE REVIEW}

Saleh Gareh et al. developed a traffic model consisting of a single and multi-lane setup using compression-based piezoelectric energy harvesting devices. The researchers studied and evaluated the prospects of utilizing the TwoDegree-of-Freedom (2DOF) electromechanical model for the application of energy foraging devices with a piezoelectric approach in roadways. Various piezoelectric materials like APC piezoelectric ceramics (APC 885) and Piezoelectric Cymbal Transducer (PCT) had been used. The researchers developed a traffic model based on the Cellular Automata (CA) model. The simulations were conducted under the noncongestion and collision-free traffic conditions, with the masses of the vehicles not differing much from that of one another. Also, the vehicles used had two axles. For the simulation of a compression-based piezoelectric traffic model, the researchers used the Java multi-threading programming language. Vibrations were imparted due to the movement of vehicles, which were then converted into electrical output using the PCTs embedded into the model. The gross output was about $35 \mathrm{~mW}$ and $20 \mathrm{~V}$ for the number of vehicles being 87 travelling at the speed of $80 \mathrm{kmph}$ within 2 minutes (single lane road). The researchers concluded that if multiple arrays of PCTs would be used, the energy that could be harvested might reach up to $170 \mathrm{~kW} / \mathrm{km}$ and could be utilized for various road electrification devices as well as communication units.

Gowtham B et al. attempted an investigation on the generation of electricity overcoming load on road pavement by performing an experiment of installing piezoelectric sensors beneath the road pavement with relative fabrication work. These helped them find appropriate positions where we could install the piezoelectric device to generate maximum efficiency. The researchers prepared a mild steel frame that acted as flexible pavement and installed the sensors beneath it. The plate at the top was connected with a spring for even distribution of the load and the sensors were installed in between two C.I. Plates to distribute the load equally. The amount of energy generated from the model was more. The project is cost-efficient as well as energy-efficient. 
Naveen Kumar and Syed Mohammad Shoaib de-signed a new model which helped in reducing traffic density on roads with higher frequency. They used piezoelectric sensors at the main junction of the road to monitor traffic frequency in order to reduce and increase the traffic flow time at peak hours. The piezoelectric setup was installed near signals and away from signals in the road pavements. As soon as the traffic density increases up to certain limits the sensors would transfer the mechanical load into electric energy and these electric currents send the signals to the traffic light through Zigbee communication. The conserved energy is only utilized for the traffic signals. This resulted in a decrease in traffic density. Also, during power cutoff, the road would still run traffic signals with the harvested energy from the piezoelectric setup.

He Zhang et al. studied and fabricated a new piezoelectric harvesting device to sort out the ungratified capabilities of the previously developed harvesters making them waterproof, electrically isolated and resistant to corrosion. The researchers also developed an analytical model revealing the effect on the efficiency of energy conversion due to embedded depth. The results state that the edge confinement has no effect on the output voltage hence not affecting the electromechanical response of the piezoelectric energy harvesters. The researchers concluded that the resultant stresses in the piezoelectric elements get dissipated with an increasing depth significantly decreasing the output voltage.

Xiaoping $\mathrm{Ji}$ et al. performed a test using a model consisting of piezoelectric ceramics. This test was used to detect the damage caused to the road pavements using smart aggregates. A composition of piezoelectric ceramics was made and enclosed with piezoelectric vibrators and epoxy resin. The model was placed beneath the road and load was applied onto it. This load was converted into electrical energy and later was used to detect the cracks within the road pavement. Whenever the thickness of the cracks increases the loadbearing capacity of the aggregate model decreases. This indicated the condition of the road through its depth.

Xuejuan Zhao et al. developed a theoretical model to harvest energy from piezoelectric pavements because of moving vehicles aiming to meet the growing demands of power through wireless electronic devices used for monitoring the structural health, traffic and also in intelligent roads. The researchers considered the pavement as a Bernoulli-Euler beam and assumed it rested on the Winkler foundation, the vehicle pressure was considered as a time-variant dynamic force and also kept the action point of the force stationary. A two-degree-of-freedom electromechanical model for energy harvesting was developed and it was tested in the laboratory. From this, the researchers evaluated that the speed of the vehicle plays a crucial role in determining the optimum resistive load along with the roughness of the road affecting the output voltage.

Hao Wang and Jingnan Zhao investigated the prospects of using the piezoelectric energy harvesters in the air-field pavements and also analyzed the fatigue cracking and rutting due to the impact of energy harvesters. With the help of the magnitude and excitation of stresses, the researchers determined the energy output. The effects of location, thickness, aircraft wheel, and aircraft loads on the energy module have also been discussed. An increase of about $24 \%$ and $42 \%$ in the power output was observed with an increase in the aircraft tire load from $154 \mathrm{kN}-172 \mathrm{kN}$ and $185 \mathrm{kN}$ respectively. Further, the behaviour of pavement where the piezoelectric energy harvesters were buried in the airfield was studied and it was observed that the shear resistance of pavement surface course needed to be enhanced at those locations.

Elham Maghsoudi Nia et al. utilized the kinetic energy of footsteps while walking as a renewable source to harvest energy and then converted it to electrical power. The researchers used piezoelectric materials in the tiles and embedded them in the pavement. A prototype was fabricated with transducers having the capabilities to store the energy produced into an electronic capacitor. The transduction and system design along with the tests conducted for optimum output has been described. Also, the whole prototype was tested by making a person weighing as per the requirement step on it and the output voltage was recorded in both series and parallel connections. A conclusion was drawn that out of the two, series and parallel connections, the parallel one gave the desired output voltage of $15 \mathrm{~V}$ generated due to the walking cycle of a human weighing $50 \mathrm{~kg}$ which was satisfactory.

Lumbumba Taty-Etienne Nyamayoka et al. highlighted 'the feasibility study of generating electric power from piezoelectric materials embedded in the asphalt layer on a highway'. The study was conducted in Pretoria, South Africa, where they aimed to achieve sustainable, economic and ecological power generation by making use of advanced technology. Certain parameters like the vehicle load, speed and loading time were assumed and based on this the average energy generated was recorded. The results when evaluated fulfilled the prime objective making the whole setup feasible. In all the energy that was generated was $1.576587613 \mathrm{kWh} / \mathrm{d}$ which was sufficient to light 6 numbers of HPS (HighPressure Sodium) street lights, each of $250 \mathrm{~W}$.

B. Al Mashaleh has researched a smart system that can generate electricity which will be generated from foot-steps. This system contains a piezoelectric device that can convert mechanical energy into electrical energy. One of the layers out of two from the generated is compressed while the other gets stretched resulting in the generation of power. This generator is connected to a self-powering circuit which collects all the energy produced and stores it in a capacitor bank. The acceptance rate of the circuit voltage ranges between $0 \mathrm{~V}$ to (+-) $500 \mathrm{~V}$ AC or DC. We can use these devices at crowded places like shopping malls, theatres, etc. which will increase power generation in relative expenses than power generation plants. 
Zhengbao Yang et al. studied the energy harvesting fundamentals and made efforts in defining and calculating the energy conversion efficiency. The energy flow in the energy harvesting system taking into consideration the energy reservoirs and energy conversion or transformation steps was analyzed theoretically. The researchers stated it necessary to consider the net energy transformation and stable state in every step of energy transformation. Other components of their theoretical analysis included electromechanically coupled models, mechanical response, electrical response, input mechanical energy and finally the efficiency. A prototype was fabricated in order to validate the efficiency which was de-fined through theoretical analysis. The parameters affecting the efficiency of piezoelectric energy harvesters were identified and analyzed quantitatively. The resonance frequency in both linear and nonlinear PEHs (Piezoelectric Energy Harvesters) was studied thoroughly. The researchers concluded that the efficiency of PEHs at their resonance state is the real working performance and it is easy to calculate.

Xiaochen $\mathrm{Xu}$ et al. studied if the utilization of piezo-electric technology in road energy harvesting was feasible. An experiment was conducted to evaluate the PZT (Lead Zirconate Titanate) generating capacity for which they used different piezoelectric materials in order to get a fair comparison between these materials regarding the generated output voltage. Also, to protect the transducers from the climatic conditions they developed a packaging box that fulfils the requirements such as spatial structure, capacity, durability, water resistance, etc. The circuit of piezoelectric transducers inside of the package was installed and the power output through cables was obtained for different loading conditions. The researchers carried out the road laying experiments where they considered two situations viz. burying in the construction period and grooving on the completed road. These situations were thoroughly studied noting the advantages and disadvantages of each.

Hongduo Zhao et al. have discussed the synergistic performance of the piezoelectric transducer and the asphalt pavement. Hence for its evaluation they made and tested an energy harvesting system that was composed of an array of transducers and an asphalt plate. The researchers observed that the transducers might be damaged due to the differential de-formation in the asphalt pavement which could be avoided by increasing the burial depth but it was seen that the efficiency decreased considerably by doing so.

Sanghoon Park et al. conducted an experiment that clarifies the effect of tensile stress electrodes on the piezo film along with the effect created due to its thickness and tried creating an energy harvester by stacking piezo films. The re-searchers applied mechanical pulse to excite the piezo films of different thicknesses up to a certain vibration amplitude maintaining the constant energy supply to check the effect of tensile stress on the films. The effect due to the thickness of the piezo film by applying higher stresses was tested and the energy harvesters by stacking the piezo films were created. The power densities for both thin and thick piezoelectric films were $81.3 \mathrm{~W} / \mathrm{m} 3$ at $4.2 \mathrm{MPa}$ and $33.8 \mathrm{~W} / \mathrm{m} 3$ at $2.2 \mathrm{MPa}$ respectively. The researchers concluded that a thin piezo film could attain higher output voltage.

Hailu Yang et al. tried improvising the service life and performance of the piezoelectric energy harvesters. The researchers designed the stacked array type of piezoelectric energy harvester along with the protection package based on the contact area of the tires. The researchers demonstrated the setup near Zhaotong City. A conclusion was drawn that the speed of vehicles and their mass affect the output voltage of the piezoelectric energy harvesters. The more the speed of the vehicle, the more voltage generated. The highest voltage generated during the demonstration was $250 \mathrm{~V}$ for an open circuit.

Thakur Aganit et al. proposed a system design for piezoelectric roads, and were located beneath the speed breakers in order to harvest energy and this energy could've been used to light the street lamps and also to the villages in its vicinity. The researchers have considered the model used in countries like Israel and the U.S.A. The model has 5 layers which contain fine gravels at the base then a thin layer of asphalt upon which a piezoelectric device was installed with quick-dry concrete after connecting them in series. Upon it came the bitumen sheet and finally the top finishes. The working of this model was in series. Firstly, the piezoelectric generators harvested mechanical energy and converted it into electrical energy. Then this energy was stored in batteries located at any one side of the road. The stored energy was then distributed as per requirements. This model helped in yielding around $44000 \mathrm{kWh}$ per year for a single lane $1 \mathrm{~km}$ road. In future, this project could be extended by installing the same device beneath speed breakers and places where vehicles are gathered daily in large numbers i.e., toll booths, drive-throughs, and parking lots of various companies.

A. T. Papagiannakis et al. studied and previewed the development of an energy harvesting system based on piezoelectric elements embedded in the pavement structure. The researchers included numerical modelling consisting of stress distribution and economic analysis of the generated power as per the given scenario of traffic. The results they evaluated propose that the piezoelectric technology could be suitable for harvesting energy from pavement structures to power the traffic lighting or any other wireless sensors without the use of any external grid power.

Ravjeet Kour and Ahmad Charif have assessed and studied the functionality of piezoelectric roads to harvest the energy produced due to vehicular motion. This research analysed various crystals that can undergo a piezoelectric effect which included natural as well as artificial crystals formed out of chemical compounds. The researchers observed that the efficiencies for the piezoelectric crystals were affected by their geometry, thickness, structure, mode of loading and fixation. The applications and limitations of this piezoelectric technology more vastly referring to the previous studies and 
projects has also been discussed. The researchers stated that this technology has a scope towards the future of energy and its sustainability.

Seonghoon Kim et al. conducted experiments to investigate the methods of energy harvesting and development of a framework along with the identification of its potential for future use. The researchers studied and compared the power generation data of various previously developed systems and used it to test and determine the economy of piezo-electric roadways. A lab experiment testing the piezoelectric asphalt materials along with concrete pavement materials was carried out. The results were evaluated based on the tests like the loaded wheel test, compression test etc. The researchers developed a framework for piezoelectric-based sensor voltage measurement.

Hongduo Zhao et al. analyzed the subtypes of Bridge-type Transducers in terms of their stiffness, frequency, output voltage. Firstly, a theoretical study on the mechanics of all the three subtypes for the axial stresses that they under-go after application of uniform static load from both the directions was conducted, also the polarity and voltage were evaluated theoretically. Based on the analyzed data, some structural alterations in the transducers for their improvement in the practical analysis were suggested. Further, the researchers compared the transducers through finite element analysis where the parameters and properties of the Bridge transducers were obtained which included the stresses and the electric potential of every subtype. Later the researchers experimented with the transducers, where the Bridge transducers were designed and fabricated and were tested based on their response to applied stress in the form of electric potential which was about $286 \mathrm{~V}$, the metal cap materials and their durability for varying thicknesses.

D. Paul and A. Roy studied piezoelectric effects from multiple projects and suggested energy harvesting methods through rigid pavements. Harvesting of electric energy is possible when a large amount of mechanical energy is applied to the piezoelectric devices. Considering this theory, they studied traffic frequency at an international airport. The piezoelectric devices can be installed under the runway and walkways. These devices act as generators and produce huge amounts of energy. The theoretical analysis concluded that the amount of energy that could be generated is enough to run a complete airport. Also use of piezoelectric devices is relatively inexpensive but efficiency can slightly be increased in further studies.

Ch Naveen Kumar focused on how to generate energy from piezoelectric harvesters and described the basic working of the piezoelectric crystals. The basic principle of piezoelectric crystals of converting mechanical energy into electrical energy is utilized to generate electricity. The author proposed two techniques out of which generating electricity from electromagnetic induced voltage is a whole new concept. This system consists of a flexible strip where one end of the strip is mounted with the piezoelectric crystals and the other end is mounted with a magnet. Whenever the vibration increases the intensity of the output of the energy generation increases. Frequency of the output increases as there are multiple sources for generating energy. The energy can be stored and utilized afterwards whenever required.

Kokkinopoulos A. et al. carried out research to explore the benefits of exploitation and usage of piezoelectric energy harvesting systems on Greek roads. The researchers studied the available technology and the factors that have an influence on energy production. The piezoelectric generator systems were implemented in Attiki Odos where the main aim was to enhance the output voltage. As the area for experimentation had high traffic density, the researchers installed the piezoelectric generators at different toll stations along the highway. Finally, the results were evaluated and it was found out that for 6 meters of road length the energy produced annually was about 22,855.217,92 kWh. After completing the experimentation, the researchers concluded that the piezoelectric system is advantageous and also there's future scope to the energy generation via piezoelectric pavements.

Xuezheng Jiang and others aimed at experimenting with a clean energy source in order to power the remote equipment that is part of road infrastructure. The researchers designed a roadway energy harvesting system which was a compressionbased system that generated energy due to compressive forces acting upon it. The transducers used in the harvester were stacked and placed close to each other for obtaining more energy output. Also, a theoretical analysis on the designed harvesting system was conducted where the coefficients and constants necessary to evaluate the energy harvesting capabilities of the harvesting system were defined. Later the researchers experimented on the designed harvesters to obtain the relationship between the electrical energy harvested and the external excitation and also verified the theoretical results with the experimental results. The researchers concluded that a single harvesting unit was able to generate $85 \mathrm{~mW}$ DC power under the excitation with 1360 $\mathrm{N}$ force amplitude and $6 \mathrm{~Hz}$ frequency. Also, the generated energy could be used for the lighting of roadside electrical equipment.

$\mathrm{H} \mathrm{J}$ Xiang et al. focused on problems that occurred during energy harvesting using piezoelectric transducers. The problems that occurred with the frequency of moving vehicles and the dependency of output were also studied, along with the different equations and formulations that don't always meet all the certain considerations. To overcome this, they designed a model which was embedded into the pavement. The researchers recorded the model's dynamic response from the pavements, and the theory of critical intensity was considered to formulate the outputs more precisely. A mathematical model was used to compute the energy harvested through the model that was measured experimentally. The behaviour of the pavement is studied by considering a model being subjected to a moving line load. 
The researchers concluded that the power generation was directly proportional to the velocity and intensity of vehicles.

Michaels W. Safer and Ephrahim Garcia identified more developed input limits to the piezoelectric material and thus increased the output power of a piezoelectric device. The researchers also compared the previously researched and designed models along with their expressions which were helpful for investigating the efficiency of the model. The researchers used a cantilever resonator type harvesting device. This device consisted of a substrate layer that was laminated on both sides (bimorph). The results showcased maximum efficiency by the proposed device comparing it with previously designed equations. A new theory had been proposed i.e., comparing the highest magnitude (predicted power) with the actual stress in the piezoelectric element. Also, the researchers recommended designing the specimen by considering two major ratios: first by length-to-width ratio, another by mass-to-cube ratio and both of them should be kept low.

Action Nechibvute et al. studied and carried out research on the renewable energy resource that was the piezoelectric microgenerators and also nanogenerators to utilize it for the wireless sensors. The researchers took into consideration every factor that influenced power generation and consumption, while also studying the storage devices and their properties. The nanogenerators were fabricated from semiconductor materials that we're able to produce both types of currents that are AC and DC. The study opened various opportunities for the development of piezoelectric micro and nanogenerators.

D. Vatansever, E. Siores and T. Shah in this paper compared harvesting of energy using piezoelectric theory and photovoltaic reaction. While using piezoelectric theory they stated the two domains of it viz. direct and converse piezoelectric effect. The researchers suggested using piezoelectric cells along with polymer material rather than using them with ceramic as they exhibit higher impact resistance and mechanical strength also the polymer material has a higher piezoelectric voltage constant. Results show better outputs using Photovoltaic reactions but they have lesser efficiency when the weather is cloudy and on monsoon days. Therefore, in countries with variable climatic conditions, using piezoelectric theory is more efficient than harvesting energy considering photovoltaic reactions.

Biqin Dong et al. presented a model to explain the non-linear phenomena of the electrical response of the specimens under large mechanical loadings. They studied the electrical responses of the cement-based piezoelectric ceramic composite when they were under mechanical loading. They combined the ceramic powders and cement uniformly to prepare the model. The results achieved were consistent with linear relationships between electrical output and mechanical input. The model was processed on excellent dynamic performance to mechanical loading.
M. Vázquez-Rodríguez et al. utilized piezoelectric materials to harvest energy from the road traffic environment and determine the technology of the semiconductor elements used in the input stage of the power harvesting system. They prepared an electrical model using piezoelectric elements excited by the mechanical action of the road traffic. The mechanical excitation was assumed equivalent to continuous real traffic and the calculations were carried out using Fourier mathematical expression method. To obtain results using this method, the design of the regulating circuit must contain a similar equivalent model. After calculation, the result guarantees the powering of a sensor's network practically.

\section{CONCLUSION}

The literature report briefs about the application of piezoelectricity in roadways as an alternative energy resource. Factors such as the material properties, experimental setup and methodology, the output voltages, result analysis and also product efficiency are the important ones in order to achieve the expected out-turn. While a lot has been done to harness the vibrational energy from the vehicles, there still seems to be a significant gap to implement this technology outside of a controlled environment. Specific to the Indian scenario this technology is non-existent. The Indian climatic conditions make this technology less susceptible for implementation on the roadways. Also, the applications of piezoelectricity have been quite limited in the case of concrete pavements. The assembly consisting of the piezoelectric transducer setup proves to be uneconomic with finite manufacturing agencies. The frequency of the amount of voltage generated is fluctuating, making it difficult for definite use. Having the potential scope for further development, the piezoelectric pavement technology can prove to be a reliable energy source in the years to come.

\section{REFERENCES}

1. Saleh Gareh, "Evaluation of Piezoelectric Energy Harvester Outcomes in Road Traffic Applications," Malaysia: IEEE Xplore, 2020

2. Xuejuan Zhao, "Piezoelectric energy harvesting from vehicles induced bending deformation in pavements considering the arrangement of harvesters," Applied Mathematical Modelling, 2020.

3. Gowtham B, "Asphalt Energy Harvesting through Piezoelectric Sensor Technology," India, 2020.

4. Naveen Kumar, "An Intelligent Traffic Light System Based on Piezo-electric Sensors,” India: IJRET, 2020.

5. He Zhang, "Piezoelectric Energy Harvesting from Roadways Based on Pavement Compatible Package," Journal of Applied Mechanics, 2019.

6. Xiaoping Ji, "Fabrication and Performance of a selfpowered damage-detection aggregate for asphalt pavement," Materials and Design, 2019.

7. Hao Wang, "Piezoelectric Energy Harvesting in Airport Pavement," New Jersey: CAIT-UTC, 2019. 
8. Elham Maghsoudi Nia, "Design of a Pavement using Piezoelectric Material," Malaysia, 2019.

9. Lumbumba Taty-Etienne Nyamayoka, "Feasibility study of embedded piezoelectric generator system on a highway for street lights electrification," South Africa, 2018.

10. Mashaleh, "Power generation using piezoelectric materials," 2018.

11.Zhenbao Yang, "On the efficiency of piezoelectric energy harvesters," Extreme Mechanics Letters, 26-37, 2017.

12.Xiaochen Xu, "Application of piezoelectric transducer in energy harvesting in pavement," Beijing: International Journal of Pavement Research and Technology, 2017.

13. Hongduo Zhao, "Synergistic performance of piezoelectric transducers and asphalt pavement," Shanghai: International Journal of Pavement Research and Technology, 2017.

14. Sanghoon Park, "Energy harvesting efficiency of piezoelectric polymer film with graphene and metal electrodes," Scientific Reports, 2017.

15. Hailu Yang, "A preliminary study on the highway piezoelectric power supply system," Beijing: International Journal of Pavement Research and Technology, 2017.

16. Aganit Thakur, "Piezoelectric Roads," Raipur: International Journal of Advance Research in Science and Engineering, 2017.

17.A T Papagiannakis, "Energy harvesting from roadways," The 6th International Conference on Sustainable Energy Information Technology (pp. 758-765), Elsevier Ltd., 2016.

18. Ravjeet Kour, "Piezoelectric roads: Energy harvesting method using piezoelectric technology," UK: Innovative Energy \& Research, 2016.

19. Seonghoon Kim, "Piezoelectric energy harvesting system assessment for highway sustainability," 52nd ASC Annual International Conference Proceedings, Associated Schools of Construction, 2016.

20. Hongduo Zhao, "Test and analysis of Bridge transducers for harvesting energy from asphalt pavement," Shanghai: International Journal of Transportation Science and Technology, 2015.

21.Debayan Paul, "Piezoelectric effect: Smart roads in green energy harvesting," Kolkata: International Journal of Engineering and Technology Research, 2015.

22.Kumar, "Energy collection via piezoelectricity," International Conference on Vibration Problems (ICOVP2015), IOP Publishing, 2015.

23. Kokkinopoulos A, "Energy harvesting implementing embedded piezoelectric generators - The potential for the Attiki Odos traffic grid," The International Conference on Technologies and Materials for Renewable Energy Environment and Sustainability, TMREES14 (pp. 10701085), Elsevier Ltd., 2014.

24. Xuezheng Jiang, "Piezoelectric energy harvesting from traffic-induced pavement vibrations," Journal of Renewable and Sustainable Energy, 2014.
25.H J Xiang, "Theoretical analysis of piezoelectric energy harvesting from traffic induced deformation of pavements," Beijing: IOP Publishing, 2013.

26. Michael W. Shafer, "The power and efficiency limits of piezoelectric energy harvesting," Journal of Vibration and Acoustics, 2013.

27.Action Nechibvute, "Piezoelectric energy harvesting devices: An alternative energy source for wireless sensors," Hindawi Publishing Corporation, 2012.

28.D Vatansever, "Alternative resources for renewable energy: Piezoelectric and photovoltaic smart structures," Global Warming - Impacts and Future Perspective, 2012.

29.Biqin Dong, "Electrical response of cement-based piezoelectric ceramic composites under mechanical loading," Hindawi Publishing Corporation, 2011.

30.M Vazquez-Rodriguez, "Modeling piezoelectric harvesting materials in road traffic applications," Mathematical Models and Methods in Modern Science, ResearchGate, 2011. 